TRANSACTIONS OF THE

AMERICAN MATHEMATICAL SOCIETY

Volume 363, Number 9, September 2011, Pages 4553-4568

S 0002-9947(2011)05132-9

Article electronically published on April 19, 2011

\title{
FINE ASYMPTOTICS OF CHRISTOFFEL FUNCTIONS FOR GENERAL MEASURES
}

\author{
ELLIOT FINDLEY
}

\begin{abstract}
Let $\mu$ be a measure on the unit circle satisfying Szegö's condition. In 1991, A. Máté calculated precisely the first-order asymptotic behavior of the sequence of Christoffel functions associated with $\mu$ when the point of evaluation lies on the circle, resolving a long-standing open problem. We extend his results to measures supported on smooth curves in the plane. In the process, we derive new asymptotic estimates for the Cesáro means of the corresponding 1-Faber polynomials and investigate some applications to orthogonal polynomials, linear ill-posed problems and the mean ergodic theorem.
\end{abstract}

\section{INTRODUCTION}

Let $\mu$ be a Borel measure of the complex plane with compact support, $\Gamma$. The so-called Christoffel functions associated with $\mu$ are defined by

$$
\lambda_{n}(\mu, z):=\inf \frac{1}{|P(z)|^{2}} \frac{1}{2 \pi} \int\left|P^{2}\right| d \mu,
$$

where the infimum is evaluated over all complex polynomials of degree at most $n-1$ which do not vanish at $z$. We want to understand how the asymptotic behavior of this sequence depends on the geometry of the support, $\Gamma$, when the point of evaluation, $z \in \Gamma$. It is easy to see, for instance, that $\lambda_{n}(\mu, z) \rightarrow \mu(\{z\})$ as $n \rightarrow \infty$ if $\Gamma$ is sufficiently regular and $z$ lies on the outer boundary of $\Gamma$. What is the rate of this convergence? For certain classes of measures supported on a circle or a union of intervals, the answer is already known to first order ([6], [12]): If $\mu$ is absolutely continuous with weight, $\mu^{\prime}$, in Szegö's class (defined below), then

$$
\lim _{n \rightarrow \infty} n \lambda_{n}\left(\mu, e^{i \theta}\right)=\frac{1}{2 \pi} \mu^{\prime}\left(e^{i \theta}\right) \quad \text { or } \quad \lim _{n \rightarrow \infty} n \lambda_{n}(\mu, x)=\pi \sqrt{1-x^{2}} \mu^{\prime}(x)
$$

(almost everywhere on the supports) when $\Gamma$ is, respectively, the unit circle or the interval, $[-1,1]$. The geometric significance of these equations is elucidated by elementary potential theory, which we briefly review.

Every measure, $\mu$, with compact support, $\Gamma$, has associated logarithmic energy defined as follows:

$$
I(\mu):=\iint \log \frac{1}{|z-w|} d \mu(z) d \mu(w) .
$$

Received by the editors October 7, 2008 and, in revised form, March 2, 2009, April 20, 2009 and May 21, 2009.

2010 Mathematics Subject Classification. Primary 30E10.

This research was partially supported by NSF grant NSF DMS 0700471.

(C)2011 American Mathematical Society Reverts to public domain 28 years from publication 
If $\Gamma$ is sufficiently dense, then $I(\nu)>\epsilon>0$ for all probability measures, where $\nu$ is supported on $\Gamma$. In this case, the fundamental theorem of potential theory guarantees a unique $\nu$, called an equilibrium measure and denoted $\nu_{\Gamma}$, whose logarithmic energy is least among all such measures. It is supported on the outer boundary of $\Gamma$ and determined entirely by the geometry of this boundary. Let $\Omega$ be the unbounded component of $\mathbf{C} \backslash \Gamma$ and $g_{\Omega}(z, \infty)$ be its Green's function with pole at infinity. If $\Gamma$ is smooth, then we may exploit the following useful representation of equilibrium measure:

$$
d \nu_{\Gamma}(z)=\frac{1}{2 \pi} \frac{\partial g_{\Omega}(z, \infty)}{\partial \mathbf{n}} d s \quad(z \in \Gamma),
$$

where $d s$ denotes arc length measure and $\mathbf{n}$, the outward normal along $\Gamma$. Recall that $g_{\Omega}(z, \infty)$ is the unique harmonic function on $\Omega$ which vanishes on the boundary, $\Gamma$, and satisfies $g_{\Omega}(z, \infty) \sim \log |z|$ as $z \rightarrow \infty$. If $\Phi$ is a conformal mapping of $\Omega$ onto the exterior of the unit disk, then, by the uniqueness of Green's function, $g_{\Omega}(z, \infty)=$ $\log |\Phi(z)|$. Thus, by equation (3i), $d \nu_{\Gamma}=(2 \pi)^{-1}\left|\Phi^{\prime}\right| d s$. For example, if $\Gamma=[-1,1]$, then $d \nu_{\Gamma}(x)=\left(\pi \sqrt{1-x^{2}}\right)^{-1} d x$. (See [8, ch. 3] for a stellar introduction.)

Equations (2) can now be consolidated:

$$
\lim _{n \rightarrow \infty} n \lambda_{n}(\mu, z)=\frac{d \mu}{d \nu_{\Gamma}}(z),
$$

where $\Gamma$ is the unit circle or interval. Since the Christoffel functions are infima, it is easy to show that the upper bound,

$$
\limsup _{n \rightarrow \infty} n \lambda_{n}(\mu, z) \leq \frac{d \mu}{d \nu_{\Gamma}}(z),
$$

holds for all finite Borel measures (a.e. on the support). (See [6, p. 435].) Until fairly recently, however, (4) was known only for continuous weights bounded away from zero. That it holds almost everywhere (with respect to $\nu_{\Gamma}$ ) for the more general class of measures satisfying Szegö's condition,

$$
\int \log \left(\frac{d \mu}{d \nu_{\Gamma}}\right) d \nu_{\Gamma}>-\infty,
$$

was a long-standing conjecture, finally proved by Máté, Nevai, and Totik in 1991 (6]). Since then, Totik has extended this result to measures supported on any compact subset of the real line that is regular with respect to the Dirichlet problem (12]).

Our main goal is to prove (4) given (6) when $\Gamma$ is a smooth Jordan curve in the Hölder space $C^{1, \alpha}$. We accomplish this by an abstraction of Máté's technique in [6] and investigate some applications. This is the first extension of these results to measures with general supports in the plane, although Totik has recently proven (4) for regular measures supported on multiple curves whose weights are continuous at $z(13])$.

Note that our method does not extend the latter formula in (2), which applies to a measure on $[-1,1]$. Indeed, no analogues of (4) have been produced for measures supported on general arcs in the plane. The sole result in this direction was proved by Golinskii for smooth weights supported on a single circular arc ([2]); his method relies on explicit asymptotics for the corresponding orthogonal polynomials. 


\section{MAIN RESULTS}

Throughout this essay, $\mathbf{C}$ denotes the complex plane, $U$ denotes a bounded, simply connected domain with boundary $\Gamma:=\partial U$ in the class $C^{1, \alpha}$, and $\Omega:=\overline{\mathbf{C}} \backslash \bar{U}$. $\Delta$ denotes the closed unit disk and $\Delta_{R}:=\{z:|z| \leq R\}$ with boundaries $\gamma$ and $\gamma_{R}$, respectively. $\Phi$ is the outer mapping function of $\Gamma$, a conformal mapping of $\Omega$ onto $\overline{\mathbf{C}} \backslash \Delta$ with $\Phi(\infty)=\infty$ and $\Psi:=\Phi^{-1}$. $\Phi^{*}$ maps $U \rightarrow \Delta$ conformally and $\Psi^{*}:=\left(\Phi^{*}\right)^{-1} . \quad \Gamma_{R}:=\Psi\left(\gamma_{R}\right)$ if $R \geq 1$ and $\Gamma_{R}:=\Psi^{*}\left(\gamma_{R}\right)$ if $R<1$. $U_{R}$ is the interior of $\Gamma_{R}$ and $\Omega_{R}:=\overline{\mathbf{C}} \backslash \overline{U_{R}}$. Let $d s$ denote arc length measure and let $d \mu=W\left|\Phi^{\prime}\right| d s+d \mu_{s}$ be a measure supported on $\Gamma$ with singular part $\mu_{s}$. Since $d \mu / d \nu_{\Gamma}=2 \pi W$ almost everywhere, the following, our main result, correctly abstracts equation (4):

Theorem 1. Let $d \mu=W\left|\Phi^{\prime}\right| d s+d \mu_{s}$ be a Borel measure supported on a closed curve $\Gamma \in C^{1, \alpha}$ for some $\alpha>0$ and assume that $W$ satisfies Szego"'s condition, (6). If $\alpha>1 / 2$, or $W$ is bounded and $d \mu_{s} \equiv 0$, then

$$
\lim _{n \rightarrow \infty} n \lambda_{n}\left(\mu, \zeta_{0}\right)=W\left(\zeta_{0}\right),
$$

for $\nu_{\Gamma}$-almost every $\zeta_{0} \in \Gamma$.

Theorem 1 is really the intersection of two broader results. Unfortunately, our proof of the upper bound, (5), requires a more stringent restriction on the smoothness of $\Gamma$ than that of the lower bound. This inadequacy is intrinsic to our method, as will become transparent in the proof.

Theorem 2. Let $d \mu=W\left|\Phi^{\prime}\right| d s+d \mu_{s}$ be a Borel measure supported on a closed curve $\Gamma \in C^{1, \alpha}$. If $\alpha>1 / 2$, or $W$ is bounded and $d \mu_{s} \equiv 0$, then

$$
\limsup _{n \rightarrow \infty} n \lambda_{n}\left(\mu, \zeta_{0}\right) \leq W\left(\zeta_{0}\right),
$$

for $\nu_{\Gamma}$-almost every $\zeta_{0} \in \Gamma$.

Theorem 3. Let $d \mu=W\left|\Phi^{\prime}\right| d s+d \mu_{s}$ be a Borel measure supported on a closed curve $\Gamma \in C^{1, \alpha}$ where $\alpha>0$. If $W$ satisfies (6), then

$$
\liminf _{n \rightarrow \infty} n \lambda_{n}\left(\mu, \zeta_{0}\right) \geq W\left(\zeta_{0}\right)
$$

for $\nu_{\Gamma^{-}}$-almost every $\zeta_{0} \in \Gamma$.

Theorem 2 admits the simpler proof, since it is an upper bound on the infimum, $\lambda_{n}$. We simply need a sequence of polynomials whose $L_{2}(\mu)$-norms converge at the optimal rate dictated by Theorem 3 , This is easy if $\Gamma$ is the unit circle. With $\zeta_{0}=e^{i \phi}$, the sequence

$$
Q_{n}(z)=\frac{1}{n} \sum_{k=0}^{n-1}\left(\overline{\zeta_{0}} z\right)^{k}
$$

suffices: $Q_{n}\left(\zeta_{0}\right)=1$ and

$$
\frac{n}{2 \pi} \int\left|Q_{n}(z)\right|^{2} d \mu(z)=\frac{1}{2 \pi} \int_{-\pi}^{\pi} \frac{1}{n}\left|\sum_{k=0}^{n-1} e^{i k(t-\phi)}\right|^{2} d \mu\left(e^{i t}\right)=\sigma_{n}(\mu, \zeta),
$$

the (order $n$ ) Fejér mean of the Fourier-Stieltjes series of $d \mu$ evaluated at $\zeta_{0}$. The sequence of Fejér means converges to $W$ for almost every $\zeta_{0}$, which proves (8). (See [14, Vol. 1, Th. III.8.1, p.105].) This is the technique of Máté and Nevai in [5. For 
a general curve, $\Gamma$, we can deduce the upper bound from this case by substituting $z^{k}$ in $Q_{n}(z)$ by the ( $k$-th order) 1-Faber polynomial associated with $\Gamma$,

$$
F_{k}(z)=\frac{1}{2 \pi i} \int_{\Gamma_{R}} \frac{\Phi^{k}(\zeta)}{\zeta-z} \Phi^{\prime}(\zeta) d \zeta \quad\left(z \in U_{R}\right) .
$$

Now, redefine $Q_{n}$ by

$$
Q_{n}(z):=\frac{1}{n} \sum_{k=0}^{n-1} F_{k}(z) \quad \text { and let } \quad S_{n}(z):=\frac{1}{n} \sum_{k=0}^{n-1} \Phi^{\prime}(z) \Phi^{k}(z) .
$$

With $d \tilde{\mu}(\theta):=d \mu\left(\Psi\left(e^{i \theta}\right)\right)$, we have

$$
\begin{aligned}
\int\left|S_{n}\right|^{2} d \mu=\int \mid & \left.\frac{1}{n} \sum_{k=0}^{n-1} \Phi(\zeta)^{k}\right|^{2}\left|\Phi^{\prime}(\zeta)\right|^{2} d \mu(\zeta) \\
& =\int_{-\pi}^{\pi}\left|\frac{1}{n} \sum_{k=0}^{n-1} e^{i k \theta}\right|^{2}\left|\Psi^{\prime}\left(e^{i \theta}\right)\right|^{-2} d \tilde{\mu}(\theta)=\frac{2 \pi}{n} \sigma_{n}\left(d \tilde{\mu} /\left|\Psi^{\prime}\right|^{2}, 1\right),
\end{aligned}
$$

so the revised sequence, $\left\{Q_{n}\right\}$, will serve our purpose if we can show that $Q_{n}-S_{n} \rightarrow$ 0 with sufficient rapidity. We can, with some restrictions on $\mu$.

Lemma 1. For any $\alpha>0$ and $\Gamma \in C^{1, \alpha}, Q_{n}-S_{n} \rightarrow 0$ pointwise and

$$
\int_{\Gamma}\left|Q_{n}-S_{n}\right|^{2} d s=o\left(n^{-1}\right) .
$$

If $\alpha>1 / 2$, then the limit holds uniformly for $z \in \Gamma$ :

$$
\sup _{z \in \Gamma}\left|Q_{n}(z)-S_{n}(z)\right|=o\left(n^{-1 / 2}\right) .
$$

Our choice of $Q_{n}$ in (12) is a natural extension of (10): The Faber polynomials generalize the monomials, $z^{k}$, in a variety of contexts, e.g. locally uniform (Taylorseries type) expansions of analytic functions in $U$. Let us quickly verify Theorem 2 using these estimates.

Proof of Theorem 2. Minkowski gives

$$
\sqrt{n}\left\|Q_{n}\right\|_{L_{2}(\mu)} \leq \sqrt{n}\left\|Q_{n}-S_{n}\right\|_{L_{2}(\mu)}+\sqrt{n}\left\|S_{n}\right\|_{L_{2}(\mu)} .
$$

Under the conditions of Theorem 2, Lemma 1 implies that $\left\|Q_{n}-S_{n}\right\|_{L_{2}(\mu)}=$ $o(1 / \sqrt{n})$, so the first term on the right-hand side vanishes as $n \rightarrow \infty$. By multiplying the outer mapping function in (12) by a constant of unit modulus, we can ensure that the Fejér means in (13) converge at $z=1$. Thus, (16) implies that

$$
\limsup _{n \rightarrow \infty} \frac{n}{2 \pi} \int\left|Q_{n}\right|^{2} d \mu \leq \lim _{n \rightarrow \infty} \frac{n}{2 \pi} \int\left|S_{n}\right|^{2} d \mu=W\left(\zeta_{0}\right)\left|\Phi^{\prime}\left(\zeta_{0}\right)\right|^{2},
$$

where $\zeta_{0}=\Psi(1)$. Finally, since $Q_{n}\left(\zeta_{0}\right)-S_{n}\left(\zeta_{0}\right) \rightarrow 0$ and $S_{n}\left(\zeta_{0}\right)=\Phi^{\prime}\left(\zeta_{0}\right)$,

$$
\limsup _{n \rightarrow \infty} \frac{1}{2 \pi} \frac{n}{\left|Q_{n}\left(\zeta_{0}\right)\right|^{2}} \int\left|Q_{n}\right|^{2} d \mu \leq W\left(\zeta_{0}\right) \text {. }
$$


The more difficult proof of the lower bound, (9), will employ Hardy space methods. $D(z)$ and $Q(z)$ represent, respectively, the outer functions associated with $\log \sqrt{W}$ in $U$ and $\Omega$. They are analytic and non-vanishing in their respective domains and have non-tangential limits almost everywhere at their common boundary, $\Gamma$, with $|D(\zeta)|^{2}=|Q(\zeta)|^{2}=W(\zeta)$, for almost every $\zeta \in \Gamma$. Fix $\zeta_{0} \in \Gamma$, a Lebesgue point of $D, Q$ and $\log W$, and choose a polynomial, $P_{n}$, of degree at most $n-1$ for which

$$
\lambda_{n}\left(\mu, \zeta_{0}\right)=\frac{1}{\left|P_{n}\left(\zeta_{0}\right)\right|^{2}} \frac{1}{2 \pi} \int\left|P_{n}\right|^{2} d \mu
$$

$P_{n}$ is defined only up to a multiplicative constant, so we may assume that the sequence $\left\{\left|P_{n}\left(\zeta_{0}\right)\right|\right\}_{n}$ is bounded above and below. For the same reason, we may assume that $\Phi\left(\zeta_{0}\right)=1$. If necessary, multiply $Q$ by a constant of unit modulus so that $Q\left(\zeta_{0}\right)=D\left(\zeta_{0}\right)$.

Máté's proof of the lower bound in [6] relies heavily on a knowledge of the locations of the zeros of $P_{n}$. Szegö proved in his canonical treatise on orthogonal polynomials that the zeros of $P_{n}$ lie on the unit circle when the measure is supported on the circle; for general supports, no analogous results exist. We can surmount this obstacle to our adaptation of Máté's method by means of a weighted BernsteinWalsh inequality, which permits an estimate of the sequence $\left\{\left|P_{n}\right|\right\}_{n}$ in vanishing neighborhoods of the point of evaluation, $\zeta_{0}$.

Lemma 2. Fix c $>0$ and assume that $W \leq 1$ satisfies Szegö's condition. $\left\{\left|P_{n}(z)\right|\right\}_{n}$ is bounded uniformly for all $\left|z-\zeta_{0}\right|<c / n$.

Before proceeding to prove the main theorem and supporting lemmata, we investigate some interesting applications.

\section{Applichtions}

Orthogonal polynomials. First we note an immediate consequence for the theory of orthogonal polynomials. Let $\left\{p_{n}(\mu, z)\right\}$ denote the sequence of orthonormal polynomials associated with the measure $\mu$. When $W$ is smooth and positive, Suetin ([11]) obtains precise norm estimates for this sequence using standard Fourieranalytic techniques. His methods fail for the more general Szegö class of measures since their weights may be highly erratic and not amenable to the methods of classical approximation theory. Although our results do not fully extend his, they do imply the following sup-norm estimate for Szegö weights:

Corollary 1. Let $\mu$ satisfy all the hypotheses of Theorem 1. For almost every $\zeta_{0} \in \Gamma$,

$$
\left|p_{n}\left(\mu, \zeta_{0}\right)\right|=o(\sqrt{n})
$$

Proof. Elementary linear algebra provides the following representation of the Christoffel functions:

$$
\lambda_{n}(\mu, z)^{-1}=\sum_{k=0}^{n-1}\left|p_{k}(\mu, z)\right|^{2} .
$$

Thus,

$$
\left|p_{n}(\mu, z)\right|^{2}=\frac{1}{\lambda_{n+1}(\mu, z)}-\frac{1}{\lambda_{n}(\mu, z)},
$$

and the result follows from Theorem 1 . 
Operator-theoretic formulation and ill-posed problems. Let $H$ be a Hilbert space with inner product (, ). Let $y \in H$ and let $A$ be a linear operator on $H$. The problem of solving the equation $A x=y$ is ill-posed if $A$ is not invertible. Illposed problems obviously have no solution in general, although stable approximate solutions minimizing $\|A x-y\|$ may be found by certain recursive algorithms. Our method pertains when $A=\zeta_{0}-T$, where $T$ is a normal operator with spectrum $\sigma(T)=\Gamma \in C^{1, \alpha}$ and $\zeta_{0} \in \Gamma$. A typical approach uses approximants of the form $x_{n}=R_{n}(T) y$, where $R_{n}$ is a polynomial of degree at most $n$. (See, for example, 11.) Ideally, $\left\|\left(\zeta_{0}-T\right) x_{n}-y\right\| \rightarrow 0$ as $n \rightarrow \infty$. What is the optimal rate of this convergence and can we find a sequence, $\left\{Q_{n}\right\}_{n}$, which attains this rate?

The answer follows immediately from Theorem[1; If $\left\{E_{\zeta}\right\}_{\zeta \in \Gamma}$ denotes the spectral family of projections associated with $T$, then

$$
\left\|\left(\zeta_{0}-T\right) x_{n}-y\right\|^{2}=\int_{\Gamma}\left|\left(\zeta_{0}-\zeta\right) R_{n}(\zeta)-1\right|^{2} d \mu_{y}(\zeta),
$$

where $d \mu_{y}(\zeta)=W_{y}(\zeta)|d \Phi(\zeta)|+d \mu_{s}(\zeta):=\left(y, d E_{\zeta} y\right)$. If $0 \notin \Gamma$, then $W_{y}$ satisfies Szegö's condition precisely when $y$ lies outside of $S_{y}:=\overline{\operatorname{span}}\left\{T^{k} y\right\}_{k \geq 1}$. Indeed, Szegö's classical result for measures $d \nu=W(\theta) d \theta$ on the unit circle is

$$
\inf _{p \in A(\Delta), p(0)=0} \int|1-p|^{2} d \nu=\exp \left(\frac{1}{2 \pi} \int \log W(\theta) d \theta\right),
$$

where $A(\Delta)$ is the set of analytic functions in $\Delta$. A conformal mapping generalizes this to any simply connected domain with boundary $\Gamma \in C^{1, \alpha}$ as long as $0 \notin$ $\Gamma$. Applying the spectral theorem as above, it follows that $y$ is isolated from $S_{y}$ precisely when $\log W_{y}$ is integrable. This gives an equivalent formulation of our main result:

Corollary 2. Let $T$ be a bounded normal operator on a Hilbert space, H, with spectrum $\Gamma \in C^{1, \alpha}$ such that $0 \notin \Gamma$. If $y \in H \backslash S_{y}$, then

$$
\liminf _{n \rightarrow \infty} n\left\|\left(\zeta_{0}-T\right) x_{n}-y\right\|^{2} \geq W_{y}\left(\zeta_{0}\right),
$$

for $\nu_{\Gamma}$-almost every $\zeta_{0} \in \Gamma$.

If the hypotheses of Theorem 2 hold and $Q_{n}(z)$ is given by (12), then $x_{n}=$ $R_{n}(T) y$ attains the optimal convergence rate as long as $\left(\zeta_{0}-z\right) R_{n}(z)-1=c Q_{n}(z)$ for some constant, $c$. When $Q_{n}\left(\zeta_{0}\right) \neq 0$, choosing $c=-1 / Q_{n}\left(\zeta_{0}\right)$ gives

$$
R_{n}(z)=\frac{1}{Q_{n}\left(\zeta_{0}\right)} \frac{Q_{n}\left(\zeta_{0}\right)-Q_{n}(z)}{\zeta_{0}-z}
$$

as an asymptotically optimal approximant, attaining the lower bound in Corollary 2. This result is relevant to ergodic theory.

Let $T_{\theta}=e^{i \theta} U$ be a rotation of a unitary operator, $U$, with full spectrum $(\sigma(U)=$ $\partial \Delta)$. The mean ergodic theorem states that

$$
\frac{1}{n} \sum_{k=0}^{n-1} T_{\theta}^{k} y \rightarrow \Pi y \quad \text { as } n \rightarrow \infty,
$$


where $\Pi$ is the orthogonal projection onto $N\left(I-T_{\theta}\right)$. In this case, $\Gamma=\partial \Delta$, so $Q_{n}$ is given by (10) with $\zeta_{0}=1$. Also, $(1-z) R_{n}(z)-1=-Q_{n}(z)$, so

$$
n\left\|\frac{1}{n} \sum_{k=0}^{n-1} T_{\theta}^{k} y\right\|^{2} \rightarrow 2 \pi W_{y}\left(e^{-i \theta}\right) \quad \text { a.e. } \theta \in[-\pi, \pi],
$$

a strengthening of the mean ergodic theorem when $y \notin S_{y}$. This implies that $y \perp N\left(I-T_{\theta}\right)$ for almost every $\theta$ and gives the precise norm-rate of convergence in (18). Corollary 2 further implies that, for almost every $\theta \in[-\pi, \pi]$, this rate cannot be improved by a different choice of polynomials, $\left\{Q_{n}(z)\right\}_{n}$.

\section{Proofs}

We begin with the proof of Lemma 2. Let $\omega_{D}$ denote harmonic measure in the domain $D$. If $\partial D$ is smooth, then there are kernels, $K_{D}(z, \zeta)$, such that $d \omega_{D}(z, \zeta)=$ $K_{D}(z, \zeta)|d \zeta|$, for $\zeta \in \partial D$. For every $r>1$, let $\Phi_{r}$ denote the conformal map of $\Omega_{r}$ onto $\mathbf{C} \backslash \Delta$ with $\Phi_{r}\left(\zeta_{0}\right)>0$ and let $\Psi_{r}$ denote its inverse. Since $\Gamma \in C^{1, \alpha}$, the Kellogg-Warschawski theorem ([7, Theorem 3.6]) states that $\Phi^{\prime}, \Psi^{\prime} \in \operatorname{Lip}(\alpha)$. Consequently, $\left\{\Phi_{r}^{\prime}\right\}$ is in $\operatorname{Lip}(\alpha)$ and has uniformly bounded Lipschitz constants for $1 / 2<r<1$. The same is true of $\Psi_{r}^{\prime}$, so the family $\left\{\Phi_{r}^{\prime}: 1 / 2<r<1\right\}$ is uniformly bounded away from 0 and $\infty$. This permits the following estimate:

Lemma 3. There is a constant $C>0$ such that for all $1 / 2<r<1, z \in \Omega_{r}$ and $\eta \in \Gamma$

$$
\int_{\Gamma_{r}} K_{U}(\zeta, \eta) K_{\Omega_{r}}(z, \zeta)|d \zeta| \leq C \frac{\left|\Phi_{r}(\eta)\right|^{2}\left|\Phi_{r}(z)\right|^{2}-1}{\left|\Phi_{r}(\eta) \overline{\Phi_{r}(z)}-1\right|^{2}} .
$$

Proof. The conformal equivalence of the harmonic measure implies that

$$
K_{\Omega_{r}}(z, \zeta)=\left|\Phi_{r}^{\prime}(\zeta)\right| K_{\mathbf{C} \backslash \Delta}\left(\Phi_{r}(z), \Phi_{r}(\zeta)\right) \leq C_{1} \frac{\left|\Phi_{r}(z)\right|^{2}-\left|\Phi_{r}(\zeta)\right|^{2}}{\left|\Phi_{r}(z)-\Phi_{r}(\zeta)\right|^{2}},
$$

for all $\zeta \in \Gamma_{r}$ and $\zeta \in \Omega_{r}$. Similarly, for every $\eta \in \Gamma$ and $z \in \Omega_{r}$,

$$
K_{U}(\zeta, \eta) \leq C_{2} \frac{1-\left|\Phi^{*}(\zeta)\right|^{2}}{\left|\Phi^{*}(\zeta)-\Phi^{*}(\eta)\right|^{2}}
$$

Note that $1-\left|\Phi^{*}(\zeta)\right|^{2} \sim \operatorname{dist}\left(\Gamma, \Gamma_{r}\right) \sim\left|\Phi_{r}(\eta)\right|^{2}-1$, uniformly for $\zeta \in \Gamma_{r}$ and $\eta \in \Gamma$, and for all $1 / 2<r<1$. This implies, since $\left|\left(\Phi^{*}\right)^{\prime}\right|$ is also bounded above and below, that

$$
K_{U}(\zeta, \eta) \leq C_{3} \frac{\left|\Phi_{r}(\eta)\right|^{2}-1}{\left|\Phi_{r}(\zeta)-\Phi_{r}(\eta)\right|^{2}}
$$

Thus, with $\Phi_{r}(\eta)=R e^{i \theta}$ and $\Phi_{r}(z)=\rho e^{i \phi}$,

$$
\begin{gathered}
\int_{\Gamma_{r}} K_{U}(\zeta, \eta) K_{\Omega_{r}}(z, \zeta)|d \zeta| \leq C_{4} \int_{\Gamma_{r}} \frac{\left|\Phi_{r}(\eta)\right|^{2}-1}{\left|\Phi_{r}(\zeta)-\Phi_{r}(\eta)\right|^{2}} \cdot \frac{\left|\Phi_{r}(z)\right|^{2}-\left|\Phi_{r}(\zeta)\right|^{2}}{\left|\Phi_{r}(z)-\Phi_{r}(\zeta)\right|^{2}}|d \zeta| \\
\leq C_{5} \int_{-\pi}^{\pi} \frac{R^{2}-1}{\left|R e^{i \theta}-e^{i t}\right|^{2}} \frac{\rho^{2}-1}{\left|\rho e^{i \phi}-e^{i t}\right|^{2}} d t .
\end{gathered}
$$

The last inequality follows again from the fact that $\Phi_{r}^{\prime}$ is uniformly bounded away from 0 . To evaluate, we use the series development of the Poisson kernel:

$$
\frac{1-x^{2}}{\left|1-x e^{i t}\right|^{2}}=\sum_{n=-\infty}^{\infty} x^{|n|} e^{i n t} \quad(0<x<1) .
$$


Since $R, \rho>1$,

$$
\begin{aligned}
& \frac{1}{2 \pi} \int_{-\pi}^{\pi} \frac{R^{2}-1}{\left|R e^{i \theta}-e^{i t}\right|^{2}} \frac{\rho^{2}-1}{\left|\rho e^{i \phi}-e^{i t}\right|^{2}} d t \\
&=\frac{1}{2 \pi} \int_{-\pi}^{\pi}\left\{\sum_{n=-\infty}^{\infty} R^{-|n|} e^{i n(t-\theta)} \times \sum_{k=-\infty}^{\infty} \rho^{-|k|} e^{i k(t-\phi)}\right\} d t \\
& \quad=\sum_{n=-\infty}^{\infty}(\rho R)^{-|n|} e^{i n(\phi-\theta)}=\frac{\rho^{2} R^{2}-1}{\left|\rho R-e^{i(\phi-\theta)}\right|^{2}},
\end{aligned}
$$

which equals the fraction on the right-hand side of (19).

Proof of Lemma 2. Let $h_{r}$ represent the solution to the Dirichlet problem in $\Omega_{r}$ with boundary data $h_{r}(\zeta)=\log |D(\zeta)|\left(\zeta \in \Gamma_{r}\right)$. First, we prove that if $r=r_{n}<1$ and $1-r_{n} \sim 1 / n$, then

$$
\left|P_{n}(z)\right| \leq C e^{-h_{r}(z)+n g_{\Omega_{r}}(z, \infty)} \quad\left(z \in \Omega_{r}\right),
$$

for some $C>0$ independent of $r$ and $n$. For $z \in \Gamma_{r}$ and $r<\rho<1$,

$$
\left|P_{n}(z) D(z)\right|^{2}=\left|\frac{1}{2 \pi} \int_{\Gamma_{\rho}} \frac{P_{n}(\zeta)^{2} D(\zeta)^{2}}{\zeta-z} d \zeta\right| \leq \frac{1}{2 \pi} \int_{\Gamma_{\rho}}\left|P_{n} D\right|^{2} d s \cdot \max _{\zeta \in \Gamma_{\rho}} \frac{1}{|\zeta-z|}
$$

Letting $\rho \rightarrow 1^{-}$, we see that

$$
\begin{aligned}
\left|P_{n}(z) D(z)\right|^{2} \leq \max _{\zeta \in \Gamma}\left|\Phi^{\prime}(\zeta)\right|^{-1} \times & \frac{1}{2 \pi} \int_{\Gamma}\left|P_{n}\right|^{2} W\left|\Phi^{\prime}\right| d s \cdot \frac{C_{1}}{1-r} \\
& \leq C_{2} \frac{\left|P_{n}\left(\zeta_{0}\right)\right|^{2} \lambda_{n}\left(\mu, \zeta_{0}\right)}{1-r} \leq C_{3} \frac{\left|P_{n}\left(\zeta_{0}\right) D\left(\zeta_{0}\right)\right|^{2}}{n(1-r)}
\end{aligned}
$$

by (17). The constant $C_{3}$ is independent of $n$ and $r$. So, $\left|P_{n}(z) D(z)\right|$ is bounded uniformly for $z \in \Gamma_{r}$ as $r=r_{n} \rightarrow 1$ as long as $1-r \sim 1 / n$. This proves, under the stated assumptions, that

$$
u(z)=\log \left|P_{n}(z)\right|+h_{r}(z)-n g_{\Omega_{r}}(z, \infty)
$$

is bounded on $\Gamma_{r}$. It is also clearly subharmonic in $\Omega_{r}$ and bounded at $\infty$, so it must be true that $u(z) \leq \log C$ in $\Omega_{r}$, which establishes (20).

Let us examine the exponent on the right-hand side of (20). Since $\Phi_{r}^{\prime}$ are uniformly bounded, we may choose $\delta>0$ so that $S_{r}:=\left\{w:\left|w-\zeta_{0}\right|<\delta \cdot \operatorname{dist}\left(\zeta_{0}, \Gamma_{r}\right)\right\}$ has $\operatorname{diam}\left(\Phi_{r}\left(S_{r}\right)\right)<\frac{1}{2} \operatorname{dist}\left(\zeta_{0}, \Gamma_{r}\right)$ for all $1 / 2<r<1$. Since $\Phi_{r}\left(\zeta_{0}\right)$ is real, this implies that the sets $\Phi_{r}\left(S_{r}\right)$ are contained in a fixed sector, $\Sigma$, of $\mathbf{C} \backslash \Delta$ emanating from 1 and symmetric about the real axis. We claim that the functions $-h_{r}$ are uniformly bounded on $S_{r}$. To see this, let $z \in S_{r}$ and apply (19) and the fact that 
$\log |D(\zeta)|^{-1}$ is harmonic in $U$ :

$$
\begin{aligned}
& -h_{r}(z)=\int_{\Gamma_{r}} \log |D(\zeta)|^{-1} K_{\Omega_{r}}(z, \zeta)|d \zeta| \\
& =\int_{\Gamma_{r}}\left(\int_{\Gamma} \log |D(\eta)|^{-1} K_{U}(\zeta, \eta)|d \eta|\right) K_{\Omega_{r}}(z, \zeta)|d \zeta| \\
& =\int_{\Gamma} \log |D(\eta)|^{-1}\left(\int_{\Gamma_{r}} K_{U}(\zeta, \eta) K_{\Omega_{r}}(z, \zeta)|d \zeta|\right)|d \eta| \\
& \leq C_{1} \int_{\Gamma} \log |D(\eta)|^{-1} \frac{\left|\Phi_{r}(\eta)\right|^{2}\left|\Phi_{r}(z)\right|^{2}-1}{\left|\Phi_{r}(\eta) \overline{\Phi_{r}(z)}-1\right|^{2}}|d \eta| .
\end{aligned}
$$

Now let $R_{r}(\theta) e^{i \theta}=\Phi_{r}(\eta)$ and set $\Phi_{r}(z)=\rho e^{i \phi}$ and $F_{r}(\theta)=\log \left|D \circ \Psi_{r}\left(R_{r}(\theta) e^{i \theta}\right)\right|^{-1}$. With a change of variables, we obtain

$$
\begin{aligned}
& -h_{r}(z) \leq C_{2} \int_{-\pi}^{\pi} F_{r}(\theta) \frac{1-1 /\left(R_{r}(\theta) \rho\right)^{2}}{\left|e^{i \theta}-e^{i \phi} /\left(R_{r}(\theta) \rho\right)\right|^{2}} d \theta \\
& \quad \leq C_{3} \frac{1}{2 \pi} \int_{-\pi}^{\pi} F_{r}(\theta) \frac{1-1 /\left(R_{r}^{\prime} \rho\right)^{2}}{\left|e^{i \theta}-e^{i \phi} /\left(R_{r}^{\prime} \rho\right)\right|^{2}} d \theta,
\end{aligned}
$$

where $R_{r}^{\prime}>1$ are constants. The second inequality follows from the fact that $R_{r}(\theta) \rho$ is bounded away from 1 uniformly for $z \in S_{r}$. But the last term is the Poisson integral, $\left[P F_{r}\right]\left(e^{i \phi} /\left(R_{r}^{\prime} \rho\right)\right)$ of $F_{r}$ evaluated at the point $e^{i \phi} /\left(R_{r}^{\prime} \rho\right)=1 / R_{r}^{\prime} \overline{\Phi_{r}(z)}$. Since $\Phi_{r}\left(S_{r}\right) \subset \Sigma$, this point is contained in the reciprocal sector, $\Sigma^{-1} \subset \Delta$. $\Sigma^{-1}$ is based at 1 and non-tangential to $\partial \Delta$, so we may apply a fundamental inequality for Poisson integrals of finite measures (see [10, p. 242, Thm. 11.20]) to conclude that

$$
-h_{r}(z) \leq C_{4}\left(M F_{r}\right)(1) \quad\left(z \in S_{r}\right)
$$

$M F_{r}$ is the Hardy maximal function of $F_{r}$ : For intervals $I$, centered at $\theta=0$, and $I^{\prime}=\left\{e^{i \theta}: \theta \in I\right\}$,

$$
\begin{aligned}
M F_{r}(1)=\sup _{I} \frac{1}{|I|} \int_{I} F_{r}(\theta) d \theta & \leq C_{5} \sup _{I} \frac{1}{|I|} \int_{\Psi_{r}\left(I^{\prime}\right)} \log |D(\eta)|^{-1}|d \eta| \\
& =C_{6} \sup _{I} \frac{\left|\Psi_{r}\left(I^{\prime}\right)\right|}{|I|} \frac{1}{\left|\Psi_{r}\left(I^{\prime}\right)\right|} \int_{\Psi_{r}\left(I^{\prime}\right)} \log W(\eta)^{-1}|d \eta|
\end{aligned}
$$

where $|\cdot|$ denotes arc-length measure. The last integral is bounded independently of $r$ since the $\operatorname{arcs} \Psi_{r}(I)$ shrink to $\zeta_{0}$, which is a Lebesgue point of $\log W$. This proves our claim.

Finally, since $z \in S_{r}$ implies that $\left|\Phi_{r}(z)\right|<1+c|1-r|, g_{\Omega_{r}}(z, \infty)=\log \left|\Phi_{r}(z)\right| \leq$ $c|1-r|$ and, therefore, $n g_{\Omega_{r}}(z, \infty)$ is bounded on $S_{r}$ as $n \rightarrow \infty$ since $1-r_{n} \sim$ $1 / n$. Therefore, (20) ensures that $P_{n}(z)$ is bounded uniformly on the sets $S_{r}$. For sufficiently small $\epsilon>0$ and large $n, r_{n}=1-1 /(n \epsilon)$ makes $\left\{z:\left|z-\zeta_{0}\right|<c / n\right\} \subset S_{r_{n}}$. This completes the proof.

Proof of Theorem 3. Since the Christoffel functions are clearly monotonic in the measure, we may presume that $W \leq 1$. Otherwise, the left-hand side of (9) is only increased. Choose a sequence $z_{n} \rightarrow 1$ non-tangentially in $\{z:|z|>1\}$ in such a 
way that $\left|z_{n}-1\right| \sim 1 / n$. We will prove that

$$
\limsup _{n \rightarrow \infty}\left\{\left|P_{n}\left(\Psi\left(z_{n}\right)\right) Q\left(\Psi\left(z_{n}\right)\right)\right|-\left|P_{n}\left(\zeta_{0}\right)\right| \sqrt{\lambda_{n}\left(\zeta_{0}\right)}\left(\sum_{k=0}^{n}\left|z_{n}\right|^{2 k}\right)^{1 / 2}\right\} \leq 0 .
$$

Let us first derive our desired result, (91), from (22). It suffices to show, for arbitrarily small $\epsilon>\epsilon^{\prime}>0$ and $\delta>0$, the existence of a sequence $z_{n} \rightarrow 1$ non-tangentially in $\{z:|z|>1\}$ and with $\epsilon^{\prime} / n<\left|z_{n}-1\right|<\epsilon / n$ such that

$$
\liminf _{n \rightarrow \infty}\left|P_{n}\left(\Psi\left(z_{n}\right)\right)\right| \geq(1-\delta)\left|P_{n}\left(\zeta_{0}\right)\right| .
$$

For then $\Psi\left(z_{n}\right) \rightarrow \zeta_{0}$ non-tangentially in $\Omega$, so that $\left|Q\left(\Psi\left(z_{n}\right)\right)\right|^{2} \rightarrow W\left(\zeta_{0}\right)$, which implies by virtue of (22) that

$$
\liminf _{n \rightarrow \infty} \lambda_{n}\left(\mu, \zeta_{0}\right) \sum_{k=0}^{n}\left|z_{n}\right|^{2 k} \geq(1-\delta)^{2} W\left(\zeta_{0}\right) .
$$

This, along with

$$
\sum_{k=0}^{n}\left|z_{n}\right|^{2 k} \leq n\left(1+\frac{\epsilon}{n}\right)^{2 n}<n e^{2 \epsilon}
$$

proves (9). Now, Lemma 2 provides constants $M$ and $c$ such that $\left|P_{n}(z)\right| \leq M$ whenever $\left|z-\zeta_{0}\right| \leq c / n$. Thus,

$$
\left|z-\zeta_{0}\right| \leq \frac{c}{2 n} \Longrightarrow\left|P_{n}^{\prime}(z)\right|=\left|\frac{1}{2 \pi i} \int_{|\zeta-z|=c /(2 n)} \frac{P_{n}(\zeta)}{(z-\zeta)^{2}} d \zeta\right| \leq \frac{2 M}{c} n,
$$

so if $\epsilon$ is sufficiently small and $\left|z-\zeta_{0}\right|<\epsilon / n$, then

$$
\left|P_{n}(z)-P_{n}\left(\zeta_{0}\right)\right|=\left|\int_{\zeta_{0}}^{z} P_{n}^{\prime}(\zeta) d \zeta\right| \leq \delta
$$

and, consequently, $\left|P_{n}(z)\right| \geq(1-\delta)\left|P_{n}\left(\zeta_{0}\right)\right|$. Since $\left|\Psi^{\prime}\right|$ is uniformly bounded, the required sequence, $\left\{z_{n}\right\}_{n}$, exists.

To establish (22), define the kernels

$$
H_{n}(z):=\sum_{k=0}^{n} z_{n}^{k} \overline{\Phi(z)}^{k}
$$

With $\widetilde{F}:=F \circ \Psi$, we have

$$
\begin{aligned}
\frac{1}{2 \pi i} \int_{\Gamma} P_{n}(\zeta) Q(\zeta) H_{n}(\zeta) \Phi^{\prime}(\zeta) d \zeta & =\frac{1}{2 \pi i} \int_{\gamma} \widetilde{P}_{n}(w) \widetilde{Q}(w) \frac{1-z_{n}^{n+1} \bar{w}^{n+1}}{1-z_{n} \bar{w}} d w \\
= & \frac{1}{2 \pi i} \int_{\gamma} \frac{\widetilde{P}_{n}(w) \widetilde{Q}(w)}{1-z_{n} \bar{w}} d w-\frac{z_{n}^{n+1}}{2 \pi i} \int_{\gamma} \frac{\widetilde{P}_{n}(w) \widetilde{Q}(w)}{w^{n}\left(w-z_{n}\right)} d w
\end{aligned}
$$

$\widetilde{P}_{n} \sim w^{n-1}$ as $w \rightarrow \infty$, so the second integral (with the substitution $z=1 / w$ ) equals

$$
\frac{z_{n}^{n}}{2 \pi i} \int_{\gamma} \frac{\widetilde{P}_{n}(1 / z) \widetilde{Q}(1 / z) z^{n-1}}{\left(z_{n}^{-1}-z\right)} d z=-z_{n} \widetilde{P}_{n}\left(z_{n}\right) \widetilde{Q}\left(z_{n}\right)
$$


Subtracting, we obtain

$$
\begin{aligned}
& z_{n} \widetilde{P}_{n}\left(z_{n}\right) \widetilde{Q}\left(z_{n}\right)+\frac{1}{2 \pi i} \int_{\Gamma} P_{n}(\zeta) Q(\zeta) H_{n}(\zeta) \Phi^{\prime}(\zeta) d \zeta \\
& =\frac{1}{2 \pi i} \int_{\gamma} \frac{\widetilde{P}_{n}(w) \widetilde{Q}(w)}{1-z_{n} \bar{w}} d w=: I .
\end{aligned}
$$

We show that $I \rightarrow 0$ as $n \rightarrow \infty$. To this end, choose a large $a>0$ and let $K_{1}=[-a / n, a / n]$ and $K_{2}=[-\pi, \pi] \backslash K_{1}$. With

$$
I_{j}:=\frac{1}{2 \pi i} \int_{K_{j}} \frac{\widetilde{P}_{n}(w) \widetilde{Q}(w)}{1-z_{n} \bar{w}} d w \quad\left(w=e^{i \theta}\right),
$$

$I=I_{1}+I_{2}$. Since $z_{n} \rightarrow 1$ non-tangentially, $\left|e^{i \theta}-z_{n}\right| \geq r \theta$, for some $r>0$. Therefore,

$$
\begin{aligned}
\left|I_{2}\right|^{2} & \leq \frac{1}{2 \pi} \int_{K_{2}}\left|\widetilde{P}_{n} \widetilde{Q}\right|^{2} d \theta \times \frac{1}{2 \pi} \int_{K_{2}} \frac{d \theta}{\left|e^{i \theta}-z_{n}\right|^{2}} \leq \frac{1}{2 \pi} \int_{\Gamma}\left|P_{n} Q\right|^{2}|d \Phi| \\
& \times \frac{1}{r^{2} \pi} \int_{a / n}^{\infty} \frac{d \theta}{\theta^{2}}=\frac{1}{2 \pi} \int_{\Gamma}\left|P_{n}\right|^{2} W\left|\Phi^{\prime}\right| d s \times \frac{n}{r^{2} \pi a}=\left|P_{n}\left(\zeta_{0}\right)\right|^{2} \lambda_{n}\left(\zeta_{0}\right) \frac{n}{\pi a r^{2}},
\end{aligned}
$$

which can be made arbitrarily small for sufficiently large $a$, since $n \lambda_{n}\left(\zeta_{0}\right)$ is bounded for all $n$, by (17). (Remember that $\left|P_{n}\left(\zeta_{0}\right)\right|$ is bounded above and below.) To estimate $I_{1}$, decompose it as $I_{1}=I_{11}+I_{12}$, where

$$
\begin{aligned}
I_{11} & :=\frac{1}{2 \pi i} \int_{K_{1}} \frac{\widetilde{P}_{n}(w) \widetilde{D}(w)}{1-z_{n} \bar{w}} d w \text { and } \\
I_{12} & :=\frac{1}{2 \pi i} \int_{K_{1}} \frac{\widetilde{P}_{n}(w)}{1-z_{n} \bar{w}}[\widetilde{Q}(w)-\widetilde{D}(w)] d w .
\end{aligned}
$$

Lemma 2 implies that

$$
\begin{aligned}
\left|I_{12}\right| & \leq \frac{1}{2 \pi} \max _{w \in K_{1}}\left|\frac{\widetilde{P}_{n}(w)}{1-z_{n} \bar{w}}\right| \times \int_{K_{1}}\left|\widetilde{Q}\left(e^{i \theta}\right)-\widetilde{D}\left(e^{i \theta}\right)\right| d \theta \\
& \leq \frac{C}{\left|z_{n}\right|-1} \int_{K_{1}}\left|\widetilde{Q}\left(e^{i \theta}\right)-\widetilde{D}\left(e^{i \theta}\right)\right| d \theta
\end{aligned}
$$

which tends to 0 as $n \rightarrow \infty$ since $n\left(\left|z_{n}\right|-1\right)>\epsilon^{\prime}>0 ; 1$ is a Lebesgue point of $\widetilde{Q}$ and $\widetilde{D}$ and $\widetilde{Q}(1)=\widetilde{D}(1)$. With $J_{1}:=\Psi\left(K_{1}\right)$, we have

$$
I_{11}=\frac{1}{2 \pi i} \int_{K_{1}} \frac{\widetilde{P}_{n}(w) \widetilde{D}(w) w}{w-z_{n}} d w=\frac{1}{2 \pi i} \int_{J_{1}} \frac{P_{n}(\zeta) D(\zeta) \Phi(\zeta)}{\Phi(\zeta)-z_{n}} \Phi^{\prime}(\zeta) d \zeta .
$$

Consider $G(z):=\Phi\left(\zeta_{0}\right)+\Phi^{\prime}\left(\zeta_{0}\right)\left(z-\zeta_{0}\right)$, the linearization of $\Phi$ about $\zeta_{0}$, and define

$$
I_{11}^{*}:=\frac{1}{2 \pi i} \int_{J_{1}} \frac{P_{n}(\zeta) D(\zeta) \Phi^{*}(\zeta)}{G(\zeta)-z_{n}}\left(\Phi^{*}\right)^{\prime}(\zeta) d \zeta .
$$

Since $\Phi \in C^{1, \alpha},|G(\zeta)-\Phi(\zeta)| \leq C_{1}\left|\zeta-\zeta_{0}\right|^{1+\alpha}$. Consequently, the curve $L:=$ $\{G(\zeta): \zeta \in \Gamma\}$ is tangent to $\gamma$ at 1 , so, since $z_{n} \rightarrow 1$ non-tangentially to $\gamma$, $\left|G(\zeta)-z_{n}\right| \geq c\left|\Phi(\zeta)-z_{n}\right| \geq c^{\prime} / n$ for all $\zeta \in \Gamma$ and sufficiently large $n$. Thus,

$$
\left|\frac{1}{\Phi(\zeta)-z_{n}}-\frac{1}{G(\zeta)-z_{n}}\right| \leq C_{2} \frac{\left|\zeta-\zeta_{0}\right|^{1+\alpha}}{n^{-2}} \leq C_{3} n^{1-\alpha}
$$


for $\zeta \in J_{1}$. By applying Cauchy's inequality, we obtain the following:

$$
\begin{gathered}
\text { (26) }\left|I_{11}-I_{11}^{*}\right| \leq \frac{1}{2 \pi}\left|\int_{J_{1}} P_{n}(\zeta) D(\zeta) \Phi(\zeta)\left(\frac{1}{\Phi(\zeta)-z_{n}}-\frac{1}{G(\zeta)-z_{n}}\right) \Phi^{\prime}(\zeta) d \zeta\right| \\
+\frac{1}{2 \pi}\left|\int_{J_{1}} \frac{P_{n}(\zeta) D(\zeta)}{G(\zeta)-z_{n}}\left[\Phi(\zeta) \Phi^{\prime}(\zeta)-\Phi^{*}(\zeta)\left(\Phi^{*}\right)^{\prime}(\zeta)\right] d \zeta\right| \\
\leq \frac{1}{2 \pi}\left(\int_{J_{1}}\left|P_{n} D\right|^{2}\left|\Phi^{\prime}\right||d \zeta|\right)^{1 / 2} \times\left(\int_{J_{1}}\left|\frac{1}{\Phi(\zeta)-z_{n}}-\frac{1}{G(\zeta)-z_{n}}\right|^{2}\left|\Phi^{\prime}(\zeta) d \zeta\right|\right)^{1 / 2} \\
+C_{4} n \frac{1}{2 \pi}\left(\int_{J_{1}}\left|P_{n} D\right|^{2}\left|\Phi^{\prime}\right||d \zeta|\right)^{1 / 2} \times\left(\int_{J_{1}} \frac{\left|\Phi(\zeta) \Phi^{\prime}(\zeta)-\Phi^{*}(\zeta)\left(\Phi^{*}\right)^{\prime}(\zeta)\right|^{2}}{\left|d \zeta \Phi^{\prime}(\zeta)\right|} \mid\right.
\end{gathered}
$$

The first term on the right-hand side of (26) is bounded above by

$$
C_{5} \sqrt{\left|P_{n}\left(\zeta_{0}\right)\right| \lambda_{n}\left(\mu, \zeta_{0}\right)} n^{1-\alpha}\left|J_{1}\right|^{1 / 2},
$$

which, by Lemma 2, converges to 0 as $n \rightarrow \infty$. ( $|\cdot|$ denotes arc-length measure.) The final term in (26) does as well, since its last integrand is continuous. Now, the integrand of $I_{11}^{*}$ is holomorphic in $U$, so we may deform the contour, $J_{1}$, to the homologous contour, $-J_{2}:=-\Psi\left(K_{2}\right)$. This gives

$$
\begin{aligned}
&\left|I_{11}^{*}\right|=\mid \frac{1}{2 \pi i} \int_{J_{2}} \frac{P_{n}(\zeta) D(\zeta) \Phi^{*}(\zeta)}{G(\zeta)-z_{n}}\left(\Phi^{*}\right)^{\prime}(\zeta) d \zeta \mid \\
& \leq \frac{1}{2 \pi}\left(\int_{\Gamma}\left|P_{n} D\right|^{2}\left|\left(\Phi^{*}\right)^{\prime}\right||d \zeta|\right)^{1 / 2} \times\left(\int_{J_{2}} \frac{\left|\left(\Phi^{*}\right)^{\prime}(\zeta)\right|}{\left|G(\zeta)-z_{n}\right|^{2}}|d \zeta|\right)^{1 / 2} \\
& \leq C_{6}\left(\int_{\Gamma}\left|P_{n} D\right|^{2}\left|\Phi^{\prime}\right||d \zeta|\right)^{1 / 2}\left(\int_{K_{2}} \frac{d \theta}{\left|e^{i \theta}-z_{n}\right|^{2}}\right)^{1 / 2} .
\end{aligned}
$$

As in (25), this can be made arbitrarily small by choosing sufficiently large $a$. We conclude that $I_{1}$ and $I_{2}$ are arbitrarily small if $n$ is sufficiently large, which establishes that $I \rightarrow 0$ as $n \rightarrow \infty$. Finally,

$$
\begin{aligned}
& \left|\frac{1}{2 \pi i} \int_{\Gamma} P_{n}(\zeta) Q(\zeta) H_{n}(\zeta) d \Phi(\zeta)\right|^{2} \\
& \quad \leq \frac{1}{2 \pi} \int_{\Gamma}\left|P_{n}(\zeta) Q(\zeta)\right|^{2}|d \Phi(\zeta)| \times \frac{1}{2 \pi} \int_{\Gamma}\left|H_{n}(\zeta)\right|^{2}|d \Phi(\zeta)| \\
& \quad=\left|P_{n}\left(\zeta_{0}\right)\right|^{2} \lambda_{n}\left(\zeta_{0}\right) \frac{1}{2 \pi} \int_{-\pi}^{\pi}\left|\sum_{k=0}^{n} z_{n}^{k} e^{-i k \theta}\right|^{2} d \theta=\left|P_{n}\left(\zeta_{0}\right)\right|^{2} \lambda_{n}\left(\zeta_{0}\right) \sum_{k=0}^{n}\left|z_{n}\right|^{2 k} .
\end{aligned}
$$

This, together with (24), completes the proof.

Proof of Lemma 1. The Laurent series $\Phi(\zeta) \sim c_{-1} \zeta+c_{0}+c_{1} / \zeta+\cdots$ converges uniformly on $\Gamma_{R}$ for sufficiently large $R$, so

$$
\frac{1}{2 \pi i} \int_{\Gamma_{R}} \frac{\Phi^{\prime}(\zeta)}{\zeta-z} d \zeta=c_{-1} \quad(z \in \Gamma),
$$

and $F_{n}(z)=I+c_{-1} \Phi^{n}(z)$, where

$$
I:=\frac{1}{2 \pi i} \int_{\Gamma_{R}} \frac{\Phi^{n}(\zeta)-\Phi^{n}(z)}{\zeta-z} \Phi^{\prime}(\zeta) d \zeta .
$$


Make the substitutions $w=e^{i t}=\Phi(\zeta)$ and $e^{i \theta}=\Phi(z)$ and factor the integrand to obtain

$$
\begin{gathered}
I=\frac{1}{2 \pi i} \int_{\gamma_{R}} \frac{w-e^{i \theta}}{\Psi(w)-\Psi\left(e^{i \theta}\right)} \sum_{k=0}^{n-1} w^{k} e^{i(n-1-k) \theta} d w \\
\quad=\frac{1}{2 \pi} \int_{-\pi}^{\pi} \frac{e^{i t}-e^{i \theta}}{\Psi\left(e^{i t}\right)-\Psi\left(e^{i \theta}\right)} \sum_{k=1}^{n} e^{i k t} e^{i(n-k) \theta} d t .
\end{gathered}
$$

(Since the latter integrand is continuous and analytic in $|w|>1$, we may deform the contour back to $\gamma$.) Now let

$$
f(x, y):=\frac{e^{i x}-e^{i y}}{\Psi\left(e^{i x}\right)-\Psi\left(e^{i y}\right)} \quad \text { and } \quad F_{y}(x):=f(x, y),
$$

and let $S_{n} F$ denote the partial sums of the Fourier series of $F$ and $D_{n}(t)$, the order$n$ Dirichlet kernel. $F_{\theta}$ is the continuation to $\gamma$ of a function analytic and bounded in $\mathbf{C} \backslash \Delta$, so its Fourier coefficients, $\hat{F}_{\theta}(k)$, with positive index vanish. Thus, from (27) we obtain

$$
\begin{aligned}
I=e^{i n \theta} \frac{1}{2 \pi} \int F_{\theta}(t) \sum_{k=1}^{n} e^{i k(t-\theta)} d t=e^{i n \theta} \int F_{\theta}(t)\left[D_{n}(t-\theta)-1\right] d t & \\
& =e^{i n \theta}\left(S_{n} F_{\theta}(\theta)-\hat{F}_{\theta}(0)\right)
\end{aligned}
$$

and, consequently, $F_{n}(z)=e^{i n \theta}\left(S_{n} F_{\theta}(\theta)-\hat{F}_{\theta}(0)+c_{-1}\right)$. In the following, the complex measures $d w /\left.w\right|_{\gamma_{R}}$ are uniformly bounded and the integrand is analytic for $|w|>1$ and continuous for $|w| \geq 1$ so we may deform the contour of integration and evaluate asymptotically: since $\Psi(w) \sim\left(c_{-1}\right)^{-1} w$ as $w \rightarrow \infty$,

$$
\hat{F}_{\theta}(0)=\frac{1}{2 \pi i} \int_{\gamma} \frac{w-e^{i \theta}}{\Psi(w)-\Psi\left(e^{i \theta}\right)} \frac{d w}{w}=\lim _{R \rightarrow \infty} \frac{1}{2 \pi i} \int_{\gamma_{R}} \frac{w}{\Psi(w)} \frac{d w}{w}=c_{-1} .
$$

Furthermore, since $F_{\theta}(\theta)=\Psi^{\prime}\left(e^{i \theta}\right)^{-1}=\Phi^{\prime}(z)$, we have

$$
F_{n}(z)-\Phi^{\prime}(z) \Phi^{n}(z)=e^{i n \theta}\left(S_{n} F_{\theta}(\theta)-F_{\theta}(\theta)\right)
$$

and so, in particular,

$$
\int\left|\sum_{k=0}^{n}\left[F_{k}(z)-\Phi^{\prime}(z) \Phi^{k}(z)\right]\right|^{2} d s \sim \int_{-\pi}^{\pi}\left|\sum_{k=0}^{n} e^{i k \theta}\left(S_{k} F_{\theta}(\theta)-F_{\theta}(\theta)\right)\right|^{2} d \theta .
$$

This reduces the problem to an estimation of the convergence $S_{n} F_{\theta} \rightarrow F_{\theta}$.

We show that the functions $F_{\theta} \in \operatorname{Lip}(\alpha)$ and have uniformly bounded Lipschitz constants. Since the $F_{\theta}$ are uniformly bounded, it suffices to prove this true of the family $\left\{1 / F_{\theta}\right\}$. Set $e^{i \theta_{k}}=u_{k}$ and $e^{i \theta}=w$. If $u_{k} \neq w$, we have

$$
\begin{gathered}
F_{\theta}\left(\theta_{1}\right)^{-1}-F_{\theta}\left(\theta_{2}\right)^{-1}=\frac{1}{u_{1}-w} \int_{w}^{u_{1}} \Psi^{\prime}(z) d z-\frac{1}{u_{2}-w} \int_{w}^{u_{2}} \Psi^{\prime}(z) d z \\
=\frac{1}{u_{1}-w} \int_{0}^{u_{1}-w}\left(\Psi^{\prime}(z+w)-\Psi^{\prime}(z / a+w)\right) d z
\end{gathered}
$$


where $a=\left(u_{1}-w\right) /\left(u_{2}-w\right)$. Thus,

$$
\begin{aligned}
\left|F_{\theta}\left(\theta_{1}\right)^{-1}-F_{\theta}\left(\theta_{2}\right)^{-1}\right| \leq \frac{C}{\left|u_{1}-w\right|} & \int_{0}^{u_{1}-w}|z-z / a|^{\alpha}|d z| \\
& \leq \frac{C^{\prime}|1-1 / a|^{\alpha}}{\left|u_{1}-w\right|}\left|u_{1}-w\right|^{1+\alpha}=C^{\prime}\left|u_{1}-u_{2}\right|^{\alpha} .
\end{aligned}
$$

If $u_{1}=w$, the result follows in a similar way. A standard result from approximation theory now applies on the rate of convergence of $\left\{S_{n}\right\}_{n}$ for functions in a Lipschitz class: $\left|S_{n} F_{\theta}(x)-F_{\theta}(x)\right| \leq C n^{-\alpha} \ln n$, where $C$ is independent of $x$ and $\theta$. (See e.g. [4. pp. 180, 192-194].) Thus,

$$
\frac{1}{n} \sum_{k=0}^{n-1}\left|S_{k} F_{\theta}(\theta)-F_{\theta}(\theta)\right| \leq \frac{C}{n} \sum_{k=0}^{n-1} \frac{\ln k}{k^{\alpha}}=O\left(\frac{\ln n}{n^{\alpha}}\right),
$$

which, with (28), proves (15) if $\alpha>1 / 2$.

To prove (14) using (29), consider the partial sums, $S_{n m}$, of the double Fourier series of $f(x, y)$. We claim that they converge to $f$ uniformly in $x$ and $y$ as $n \sim$ $m \rightarrow \infty$. Let $D_{n}(t)$ denote the (normalized) Dirichlet kernels:

$$
\begin{gathered}
S_{n m}(x, y)-f(x, y)=\iint D_{n}(t) D_{m}(u)[f(x-t, y-u)-f(x, y)] d t d u \\
=\iint D_{n}(t) D_{m}(u)[f(x-t, y-u)-f(x-t, y)] d t d u \\
\quad+\int D_{n}(t)[f(x-t, y)-f(x, y)] d t=: I_{1}+I_{2} .
\end{gathered}
$$

We have already shown that $\left|I_{2}\right|=O\left(n^{-\alpha} \ln n\right)$. On the other hand, since $F_{x}(y)=$ $F_{y}(x)$

$$
\begin{aligned}
&\left|I_{1}\right| \leq \sup _{t} \mid \int D_{m}(u)[f(x-t,y-u)-f(x-t, y)] d u\left|\times \int\right| D_{n}(\tau) \mid d \tau \\
&=\sup _{t}\left|S_{m} F_{x-t}(y)-F_{x-t}(y)\right| \ln n \leq C \frac{\ln n \ln m}{m^{\alpha}} .
\end{aligned}
$$

This proves our claim.

We may now evaluate the right-hand side of (29) in terms of the development

$$
\frac{w-u}{\Psi(w)-\Psi(u)} \sim \sum_{m, l} \frac{c_{m l}}{w^{m} u^{l}}:=\lim _{N \rightarrow \infty} \sum_{m, l=0}^{N} \frac{c_{m l}}{w^{m} u^{l}} \quad(|w| \geq 1,|u| \geq 1),
$$

which converges uniformly on the torus $\gamma \times \gamma$ :

$$
S_{k} F_{\theta}(\theta)=\sum_{|j|<k} \frac{1}{2 \pi} \int_{-\pi}^{\pi} e^{i j(\theta-t)} \sum_{m, l} c_{m l} e^{-i(m \theta+l t)} d t=\sum_{j=0}^{k} \sum_{m=0}^{\infty} c_{m, j} e^{-i(m+j) \theta}
$$


Also, $F_{\theta}(\theta)=\sum_{m, l} c_{m l} e^{-i(m+l) \theta}$, so

$$
\begin{aligned}
& \frac{1}{2 \pi} \int_{-\pi}^{\pi}\left|\sum_{k=0}^{n} e^{i k \theta}\left(S_{k} F_{\theta}(\theta)-F_{\theta}(\theta)\right)\right|^{2} d \theta \\
& =\frac{1}{2 \pi} \int_{-\pi}^{\pi}\left|\sum_{k=0}^{n} \sum_{j>k} \sum_{m \geq 0} c_{m j} e^{-i(m+j-k) \theta}\right|^{2} d \theta=\sum_{k=0}^{n} \sum_{r=j-k}^{\infty} \sum_{j>k}\left|c_{r+k-j, j}\right|^{2} \\
& =\sum_{k=0}^{n} \sum_{r=l}^{\infty} \sum_{l=1}^{\infty}\left|c_{r-l, l+k}\right|^{2}=\sum_{k=0}^{\infty} \sum_{r=0}^{\infty} \sum_{l=1}^{\infty}\left|c_{r, l+k}\right|^{2}
\end{aligned}
$$

These series converge since the continuity of $f$ implies that

$$
\sum_{r, l}\left|c_{r, l}\right|^{2}=\frac{1}{4 \pi^{2}} \iint|f(x, y)|^{2} d x d y<\infty .
$$

Consequently,

$$
\frac{1}{n} \sum_{k=0}^{n} \sum_{r, l}\left|c_{r, l+k}\right|^{2} \rightarrow 0 \quad(n \rightarrow \infty),
$$

which, by virtue of (29) and (30), completes the proof.

\section{ACKNOWLEDGMENTS}

The author would like to thank Vilmos Totik for his pivotal contributions to this work. Lemma 2, in particular, was proposed and ultimately proved by him, although a somewhat different proof is given here.

\section{REFERENCES}

[1] A. Bakushinsky and A. Goncharsky. Ill-Posed Problems: Theory and Applications. Kluwer Academic Publishers, Boston, 1994. MR 1325921 (96d:65101)

[2] L. Golinskii. The Christoffel Function for Orthogonal Polynomials on a Circular Arc. J. Approx. Theory 101, pp. 165-174 (1999). MR1726450(2001b:42032)

[3] U. Grenander and G. Szegö. Toeplitz Forms and their Applications. University of California Press, Los Angeles, 1958. MR0094840(20:1349)

[4] N. Korneichuk. Exact Constants in Approximation Theory. Cambridge University Press, New York, 1991. MR 1124406 (92m:41002)

[5] A. Máté and P. Nevai. Bernstein's inequality in $L^{p}$ for $0<p<1$ and $(C, 1)$ bounds for orthogonal polynomials, Ann. of Math. (2) 111 (1980), 145-154. MR558399 (81c:42003)

[6] A. Máté, P. Nevai and V. Totik. Szegö's Extremum Problem on the Unit Cricle, Ann. of Math. (2) 134 (1991), 433-453. MR.1127481 (92i:42014)

[7] Ch. Pommerenke. Boundary Behavior of Conformal Mappings, Grundlehren der mathematischen Wissenschaften, 299, Springer-Verlag, Berlin, Heidelberg, New York, 1992. MR.1217706 (95b:30008)

[8] T. Ransford. Potential Theory in the Complex Plane. Cambridge University Press, Cambridge, 1995. MR1334766 (96e:31001)

[9] W. Ross and W. Wogen. Common Cyclic Vectors for Normal Operators, Ind. Univ. Math. J. 53 (2004), 1537-1550. MR2106335 (2005h:47041)

[10] W. Rudin. Real and Complex Analysis, 3rd ed., Mc-Graw Hill, Singapore, 1987. MR924157 (88k:00002)

[11] P. K. Suetin. Fundamental Properties of Polynomials Orthogonal on a Contour. Russian Math. Surveys 21 (1968), 35-83. MR0198111 (33:6270)

[12] V. Totik. Asymptotics for Christoffel functions for general measures on the real line. J. Anal. Math. 81 (2000), 283-303. MR1785285 (2001j:42021) 
[13] V. Totik. Christoffel functions on curves and domains. Trans. Amer. Math. Soc. 362 (2010), 2053-2087. MR 2574887 (2011b:30006)

[14] A. Zygmund. Trigonometric Series, Vols. I and II, 2nd ed., Cambridge Univ. Press, Cambridge, 1979. MR0236587 (38:4882)

E-mail address: marty0801@gmail.com 